\title{
Immunotherapy utilization for hepatobiliary cancer in the United States: disparities among patients with different socioeconomic status
}

\author{
Kota Sahara $^{1,2}$, S. Ayesha Farooq ${ }^{1}$, Diamantis I. Tsilimigras ${ }^{1}$, Katiuscha Merath ${ }^{1}$, Anghela Z. Paredes ${ }^{1}$, \\ Lu Wu ${ }^{1}$, Rittal Mehta ${ }^{1}$, J. Madison Hyer ${ }^{1}$, Itaru Endo ${ }^{2}$, Timothy M. Pawlik ${ }^{1}$ \\ ${ }^{1}$ Department of Surgery, Division of Surgical Oncology, The Ohio State University Wexner Medical Center and James Comprehensive Cancer \\ Center, Columbus, OH, USA; ${ }^{2}$ Gastroenterological Surgery Division, Yokohama City University School of Medicine, Yokohama, Japan \\ Contributions: (I) Conception and design: K Sahara, DI Tsilimigras; (II) Administrative support: I Endo, TM Pawlik; (III) Provision of study material \\ or patients: K Sahara, K Merath, AZ Paredes; (IV) Collection and assembly of data: K Sahara, SA Farooq, L Wu; (V) Data analysis and interpretation: \\ SA Farooq, JM Hyer, R Mehta; (VI) Manuscript writing: All authors; (VII) Final approval of manuscript: All authors. \\ Correspondence to: Timothy M. Pawlik, MD, MPH, PhD, FACS, FRACS (Hon.). Professor and Chair, Department of Surgery; The Urban Meyer III \\ and Shelley Meyer Chair for Cancer Research; Professor of Surgery, Oncology, Health Services Management and Policy; The Ohio State University, \\ Wexner Medical Center, 395 W. 12th Ave., Suite 670, Columbus, OH, USA. Email: Tim.Pawlik@osumc.edu.
}

\begin{abstract}
Background: Patients with advanced hepatobiliary cancer (HBC) have a dismal prognosis and limited treatment options. Immunotherapy has been considered as a promising treatment, especially for cancers not amenable to surgery.

Methods: Between 2004, and 2015, patients diagnosed with hepatocellular carcinoma (HCC), intra- and extrahepatic cholangiocarcinoma and gallbladder cancer (GBC) were identified in the National Cancer Database.

Results: Among 249,913 patients with HBC, only 585 (0.2\%) patients received immunotherapy. Among patients who received immunotherapy, most patients were diagnosed between 2012 and 2015, had private insurance, as well as an income $\geq \$ 46,000$ and were treated at an academic facility. The use of immunotherapy among HBC patients varied by diagnosis (HCC, 67.7\%; bile duct cancer, 14\%). On multivariable analysis, a more recent period of diagnosis (OR 1.80, 95\% CI: 1.44-2.25), median income >\$46,000 (OR 1.43, 95\% CI: 1.11-1.87), and higher tumor stage (stage III, OR 2.22, 95\% CI: 1.65-3.01; stage IV, OR 3.24, 95\% CI: 2.41-4.34) were associated with greater odds of receiving immunotherapy.

Conclusions: Overall utilization of immunotherapy in the US among patients with HBC was very low, yet has increased over time. Certain socioeconomic factors were associated with an increased likely of receiving immunotherapy, suggesting disparities in access of patients with lower socioeconomic status.
\end{abstract}

Keywords: Immunotherapy; hepatobiliary cancer (HBC); trends; socioeconomic status

Submitted Apr 22, 2019. Accepted for publication Jun 24, 2019.

doi: 10.21037/hbsn.2019.07.01

View this article at: http://dx.doi.org/10.21037/hbsn.2019.07.01

\section{Introduction}

Hepatobiliary cancer (HBC) consists of primary liver malignancies including hepatocellular carcinoma (HCC), gallbladder cancer (GBC), as well as cholangiocarcinoma (1-3). In the United States (US), the incidence of HCC and biliary tract cancers has been steadily increasing due to the high prevalence of chronic hepatitis and the epidemic of nonalcoholic fatty liver disease (4). In fact, according to a Surveillance, Epidemiology, and End Results (SEER) analysis, there were an estimated 50,650 new cases of HBC in 2016 resulting in 30,880 deaths (5). Although surgery can be potentially curative for patients with early stage disease, 
patients with advanced HBC disease generally have a dismal prognosis with an expected survival of less than 1 year $(6,7)$. In addition, treatment options are often limited for patients with unresectable or recurrent HBC disease. Specifically, gemcitabine, cisplatin, and sorafenib, an oral tyrosine kinase inhibitor, are the agents most often employed in the setting of advanced HBC disease (7).

Given the current limited options in the treatment of advanced HBC, immunotherapy has been considered a promising treatment option, especially for advanced cancers not amenable to surgical resection (8). Immunotherapy has demonstrated efficacy in the treatment of cancers such as hematological malignancies, melanoma, and lung cancer in several phase III trials (9). In 2011 the Federal Drug Administration (FDA) approved ipilimumab, a human cytotoxic T-lymphocyte antigen 4 (CTLA-4)blocking antibody, while in 2014-2015 nivolumab, a programmed death 1 (PD-1) inhibitor, was approved as a new immunotherapy application in patients with advanced HBC $(10,11)$. In turn, an increasing number of clinical trials are currently evaluating the efficacy of different immunotherapeutic regimens in the treatment of advanced HBC (12-14). Overall utilization of immunotherapy among patients diagnosed with $\mathrm{HBC}$ has not been previously defined, however. In addition, the characteristics and factors associated with receipt of immunotherapy among HBC patients have not been examined. As such, the objective of the current study was to assess the nationwide utilization of immunotherapy in the treatment of HBC, as well as examine socioeconomic and disease-related factors that might be associated with the receipt of immunotherapy among patients treated for HBC in the US.

\section{Methods}

\section{Study population and data collection}

Patients who had a diagnosis of HCC, ICC, GBC, or extrahepatic bile duct cancer between January 1, 2004 and December 31, 2015 were identified from the National Cancer Database (NCDB) and were included in the current study. Patients with unknown immunotherapy and/or surgery status were excluded from the analytic cohort. Furthermore, patients with premalignant lesions, neuroendocrine neoplasms, mesenchymal tumors, secondary tumors or American Joint Committee on Cancer (AJCC) clinical Stage 0 tumors were also excluded.

The NCDB is a joint venture of the American College of Surgeons and the American Cancer Society. The NCDB consists of 34 million records collected from 1,500 hospitals nationally that represents $70 \%$ of the new oncology cases in the US. The NCDB provides data on demographic characteristics such as age, sex, race, insurance status, income, as well as facility location and type. The NCDB also provides information such as the Charlson-Deyo comorbidity score (CCS), tumor grade, clinical stage, as well as treatment modalities such as chemotherapy, radiation, surgery, and immunotherapy. Patients were identified using the appropriate International Classification of Diseases for Oncology, $3^{\text {rd }}$ edition (ICD-O-3) codes for histology and primary site (Table S1).

\section{Statistical analysis}

Descriptive statistics were presented as median values with the inner quartile range for continuous variables and frequency (\%) for categorical variables. Chi-squared tests and Wilcoxon rank-sum test were performed to assess the possible association of categorical and continuous variables, respectively, to receipt of immunotherapy. All variables that were associated with receipt of immunotherapy on bivariate analysis were entered into the multivariable logistic regression analysis. A backwards stepwise model section approach was used to define the final model. To assess discrimination and model goodness-of-fit, the concordance (C) statistic and Hosmer-Lemeshow goodness-of-fit statistic were calculated. Statistical significance was assessed at $\alpha=0.05$. All analyses were performed using SAS software v.9.4. (SAS Institute Inc., Cary, NC, USA).

\section{Results}

\section{Characteristics of patients who received immunotherapy}

Among the 249,913 patients diagnosed with HBC who met inclusion criteria, only a minority received immunotherapy $(\mathrm{n}=585,0.2 \%)$ (Table 1) (Figure 1). Among patients who received immunotherapy, most patients were Caucasian $(\mathrm{n}=427,73.0 \%)$, had a CCS of $0(\mathrm{n}=364,62.2 \%)$, were diagnosed between 2012 and 2015 ( $\mathrm{n}=272,46.5 \%)$, as well as had private insurance $(\mathrm{n}=273,46.7 \%)$ and an income $\geq \$ 46,000(\mathrm{n}=242,41.4 \%)$. In addition, most patients who received immunotherapy were treated at an academic facility $(\mathrm{n}=319,54.5 \%)$. The use of immunotherapy among HBC patients varied by diagnosis (HCC $n=396,67.7 \%$; bile duct cancer $n=82,14.0 \%)$. The proportion of stage 
Table 1 Demographics and characteristics stratified by the utilization of immunotherapy

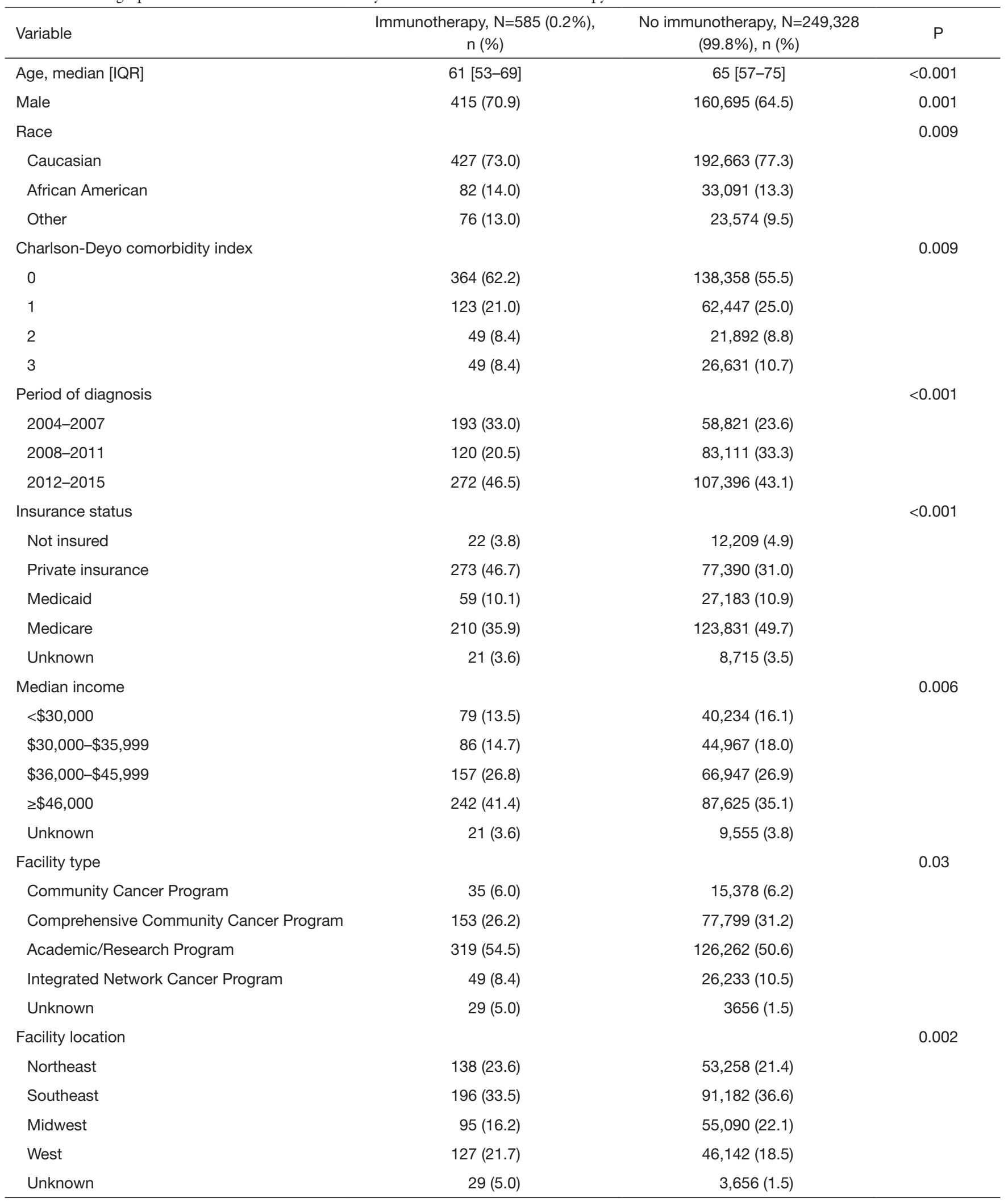

Table 1 (continued) 
Table 1 (continued)

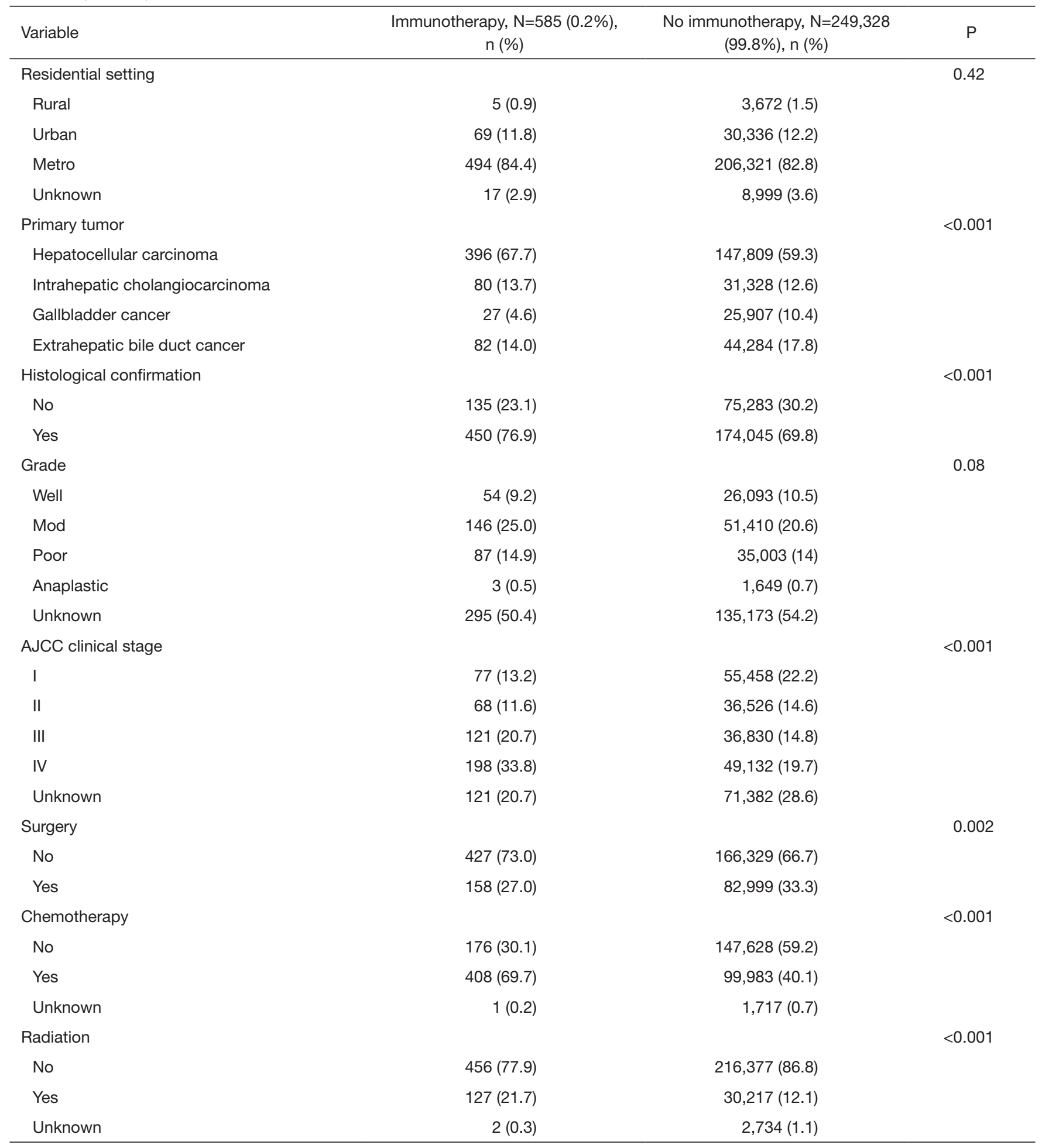

IQR, interquartile range; well, well differentiated; mod, moderately differentiated; poor, poorly differentiated. 


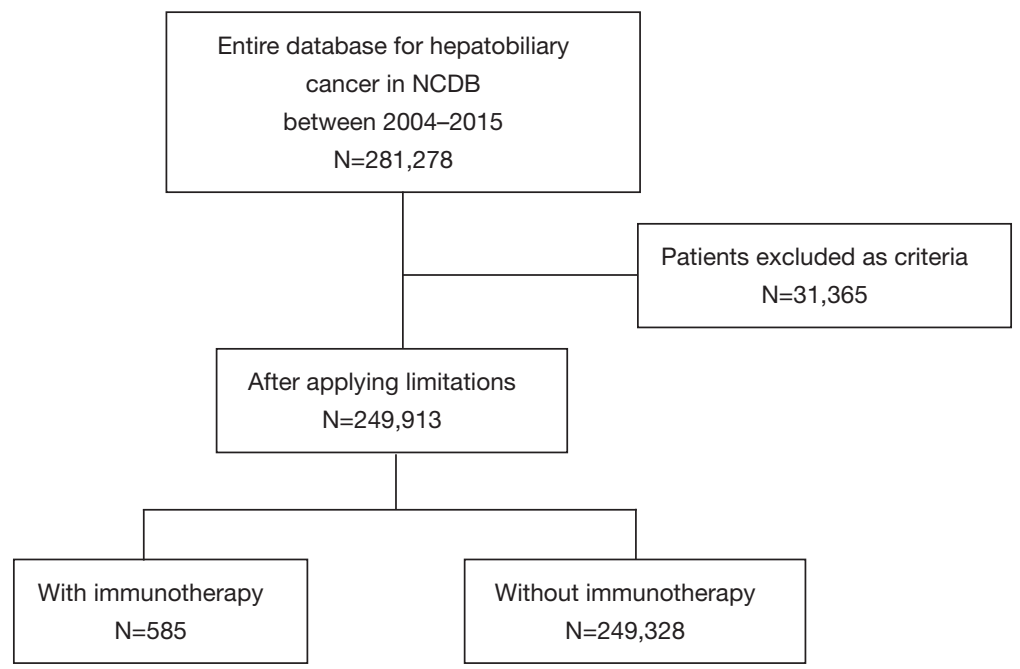

Figure 1 Flow diagram of the study sample selection.

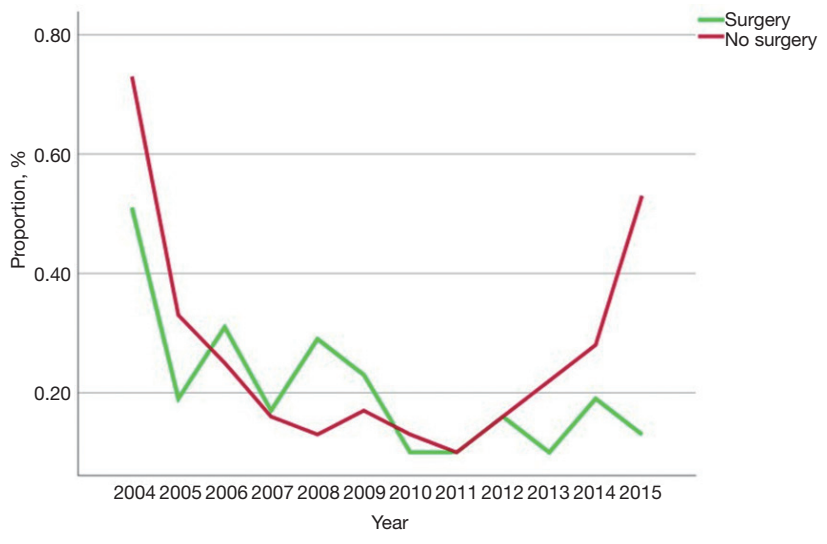

Figure 2 Utilization of immunotherapy over time stratified among patients who did and did have surgery.

III and IV disease was also higher among patients who did versus did not receive immunotherapy (54.5\% vs. $34.5 \%$, respectively; $\mathrm{P}<0.001)$. Most $\mathrm{HBC}$ patients who received immunotherapy had also received traditional cytotoxic chemotherapy ( $\mathrm{n}=408,69.7 \%)$, whereas a smaller number of patients had undergone radiation therapy $(\mathrm{n}=127,21.7 \%)$ or had surgical resection $(\mathrm{n}=158,27 \%)$. Among nonsurgical patients, a biphasic trend was observed with regard to overall immunotherapy utilization; specifically, over the years examined there was a decline in immunotherapy utilization until 2011 with a subsequent increase after 2012 peaking in 2015. In contrast, the utilization of immunotherapy among patients who had surgical resection for HBC steadily decreased over time (Figure 2).

\section{Pathological characteristics after surgery relative to the timing of immunotherapy}

Among the 136 patients who received surgery and immunotherapy and had available data on the timing of immunotherapy relative to the surgical episode, 31 (22.8\%) patients received neoadjuvant immunotherapy and 105 (77.2\%) received adjuvant immunotherapy (Table 2). The vast majority of patients with neoadjuvant immunotherapy had a diagnosis of HCC $(n=26,83.9 \%)$. Similarly, most patients who received adjuvant immunotherapy had HCC ( $\mathrm{n}=51,48.6 \%)$, followed by extrahepatic bile duct cancer ( $\mathrm{n}=34,32.4 \%)$. Among surgical patients receiving immunotherapy, 17 (12.5\%) had N1/N2 disease and 13 (9.6\%) had M1 disease. Most patients who received neoadjuvant $(\mathrm{n}=22,71.0 \%)$ and adjuvant immunotherapy $(65,61.9 \%)$ had an R0 margin resection.

\section{Timing and factors associated with immunotherapy receipt}

Distribution plots with the y-axis defined as the probability density function for the kernel density estimation associated with receipt of immunotherapy were examined. Specifically, the timing of immunotherapy receipt among patients treated for HBC was graphically displayed based on the number of days from diagnosis to receipt of immunotherapy among surgical and non-surgical patients. Among patients who underwent surgical resection for HBC, immunotherapy was more likely to be administered at a mean 96.8 days after diagnosis (post-surgery, mean 50.6 days; post- 
Table 2 Pathological characteristics among patients undergoing surgery stratified by the timing of immunotherapy

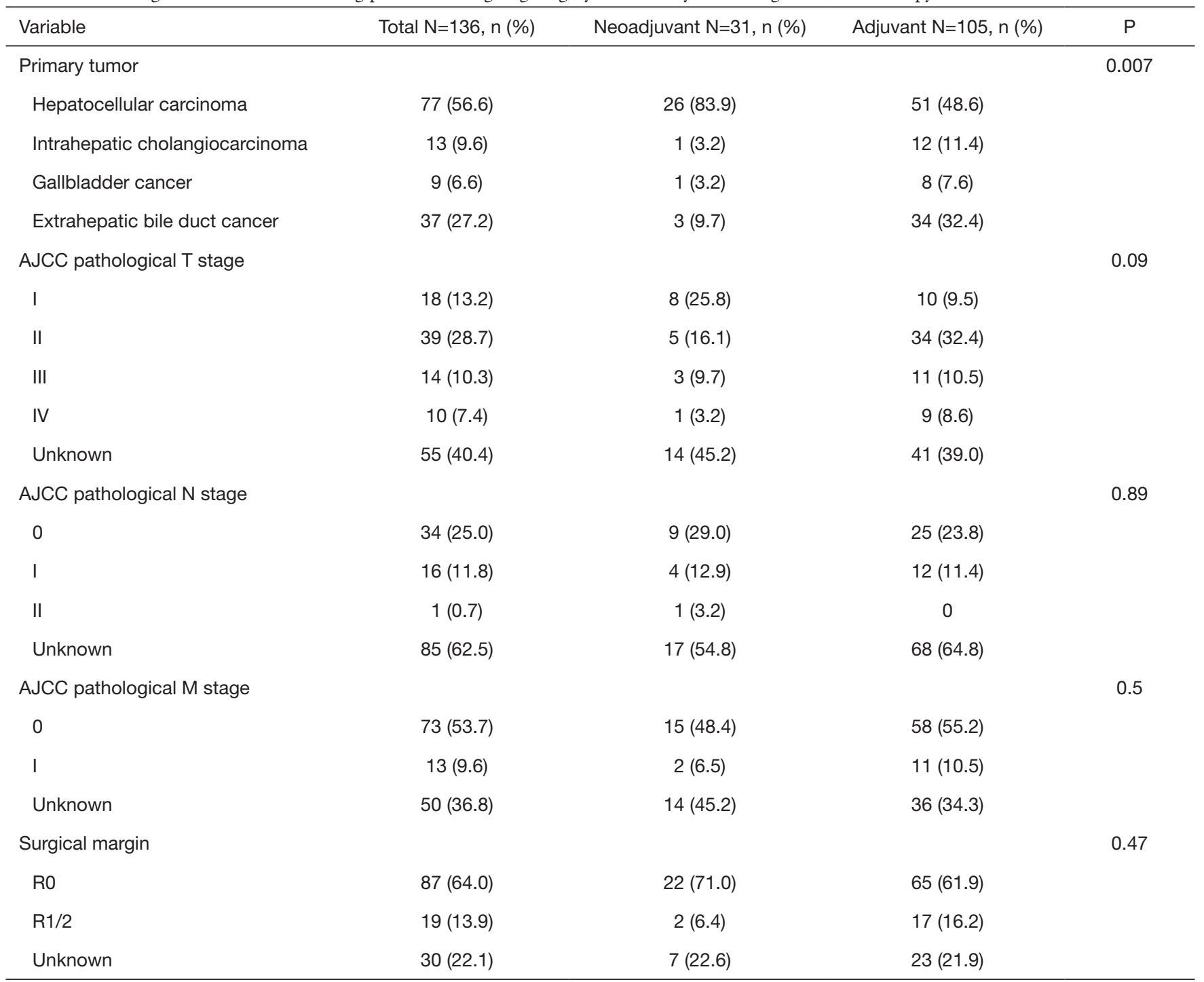

cytotoxic chemotherapy, mean 76.8 days; post-radiation, mean 108.1 days) (Figure 3A). Among patients who did not undergo a surgical procedure for HBC, immunotherapy was administered a mean of 73.1 days from diagnosis (postcytotoxic chemotherapy, mean 54.5 days; post-radiation, mean 74.7 days) (Figure 3B). Among the 427 patients who received immunotherapy and did not undergo surgery, 257 patients also had another type of systemic chemotherapy [prior immunotherapy $\mathrm{n}=30(11.7 \%)$, concurrent with other chemotherapy $n=131(51.0 \%)$, and after other chemotherapy $n=96(37.3 \%)]$.

On multivariable analysis, several socioeconomic and clinical factors were associated with the receipt of immunotherapy (Table 3). In particular, younger patient age [referent $<65$ years: $\geq 65$ years, odds ratio (OR) $0.69,95 \%$ confidence interval (CI): 0.58-0.82], a more recent period of diagnosis (referent 2008-2011: 2012-2015, OR 1.80, $95 \%$ CI: $1.44-2.25$ ), median income $\geq \$ 46,000$ (referent $<\$ 30,000$ : OR 1.43, 95\% CI: 1.11-1.87), diagnosis of HCC (referent: GBC, OR: 2.54, 95\% CI: 1.70-3.79), ICC (OR: 1.97, 95\% CI: 1.26-3.08) and extrahepatic bile duct cancer (OR: 1.68, 95\% CI: 1.08-2.61), higher tumor stage (referent stage I: stage III, OR 2.22, 95\% CI: 1.65-3.01; stage IV, OR 3.24, 95\% CI: 2.41-4.34) and prior receipt of cytotoxic chemotherapy (OR 3.36, 95\% CI: 2.79-4.04) were associated with a higher likelihood of receiving 

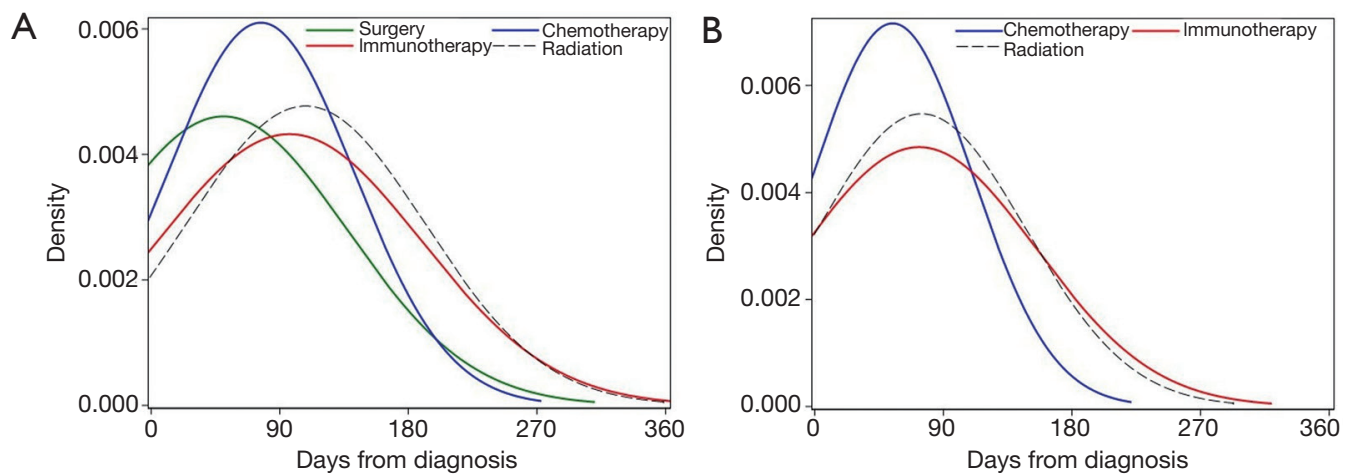

Figure 3 Distribution plots of days from diagnosis for immunotherapy and other therapies among the patients with (A) and without surgery (B) in entire cohort.

immunotherapy. Other variables such as gender, insurance status, facility type, residential setting, tumor grade, as well as history of surgical resection or radiation therapy were not associated with the odds of receiving immunotherapy.

\section{Discussion}

Recent data have suggested that chronic inflammation may contribute to the risk of developing certain solid tumors types (15). HBC, especially HCC, have been associated with chronic inflammation such as viral hepatitis and nonalcoholic or alcoholic steatohepatitis (16). The oncogenesis of HBC in this setting creates a unique 'tumor microenvironment' that leads to increased immune evasion and T-cell exhaustion (17). In fact, HCC has been reported to demonstrate high levels of PD-1 expression and immunosuppressive cytokines, suggesting that immunotherapeutic approaches may be useful in the treatment of HBC $(15,17-20)$. While progress has been made in immune-based approaches in the treatment of various other malignancies including melanoma, nonsmall cell lung cancer (NSCLC), renal and bladder cancer (21-24), immunotherapy has only recently been considered as a therapeutic option for patients with advanced HBC (16). The utilization of immunotherapy has not been studied and data on use of immunotherapy among patients with HBC in the US remain largely unknown. The current study was important because we assessed the overall utilization of immunotherapy among $\mathrm{HBC}$ patients, examined temporal trends in the utilization, as well as defined the factors associated with receipt of immunotherapy. Of note, only a very small minority $(0.2 \%)$ of patients received immunotherapy for $\mathrm{HBC}$ with the most common indication being HCC. Perhaps not surprisingly, the majority of patients who received immunotherapy were not surgical candidates (73\%), had stage III and IV disease (54.5\%) at the time of presentation, and most patients received immunotherapy late in the course of their disease. While receipt of immunotherapy was not associated with insurance status, it was associated with median income and a more recent period of diagnosis.

Several clinical trials are currently evaluating the efficacy of different immunotherapeutic regimens in HBC, including peptide-based vaccines, dendritic cell (DC)based vaccines and antibodies (25). One phase I/II trial (CheckMate 040) demonstrated that the PD-1 inhibitor nivolumab had an acceptable safety profile and promising efficacy among patients with advanced HCC (26). Based on the results of this trial, the FDA approved nivolumab for the treatment of patients with advanced or metastatic HCC who have previously been treated with sorafenib $(16,26)$. As such, it was not surprising in the current study that most patients who received immunotherapy had a diagnosis of HCC relative to other cancers such as gallbladder or cholangiocarcinoma. However, as the indications for immunotherapy for HBC expand, the number of cancer immunotherapy trials listed on the US National Institutes of Health trial registry for all HBC tumor types has progressively increased over the years (27). While the overall use of immunotherapy was very low in the years examined, we did demonstrate an increased trend in the use of immunotherapy over time especially among patients who were not surgical candidates-with the biggest increase after 2015 (Figure 2). In fact, a more recent period of diagnosis was associated with an $80 \%$ increased likelihood of immunotherapy receipt (OR 1.80, 95\% CI: 1.44-2.25).

Several factors may influence the availability and 
Table 3 Bivariable and multivariable analysis of factors associated with receipt of immunotherapy

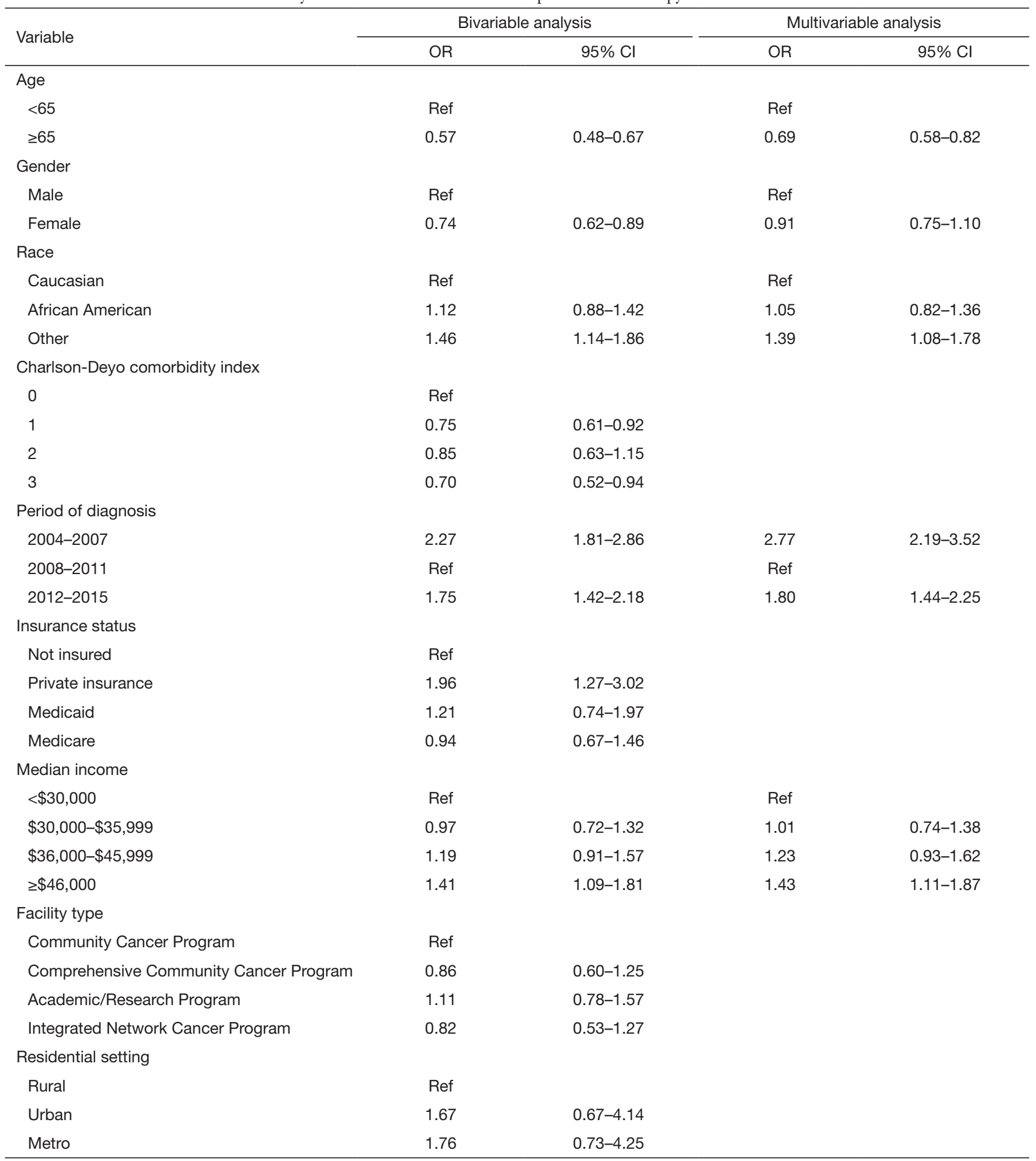

Table 3 (continued) 
Table 3 (continued)

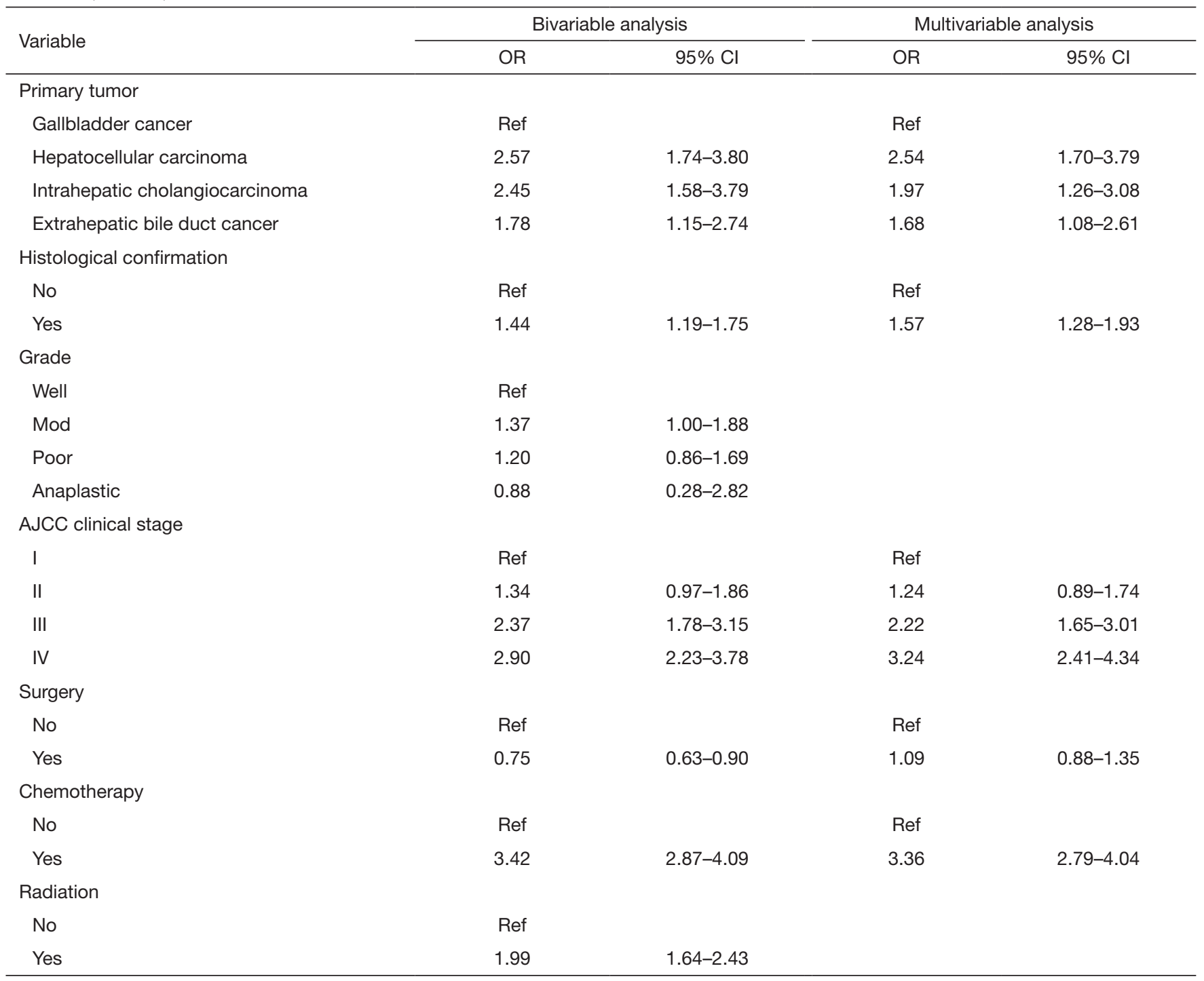

Well, well differentiated; mod, moderately differentiated; poor, poorly differentiated.

quality of cancer care. Structural factors such as health insurance or geographical distance, patient factors such as race, ethnicity, socioeconomic status, stage of disease, as well as other factors such as patient/physician preferences may all impact possible receipt of cancer therapies (28). Ward et al. suggested that although many factors contribute to treatment differences, unequal access to health care for financial or economic reasons may be the most important factor (29). To this point, in the current study, immunotherapy utilization for HBC varied among patients based on socioeconomic status. Specifically, after adjusting for competing risk factors such as tumor stage, higher income patients remained considerably more likely to receive immunotherapy compared with lower income individuals (OR 1.43, 95\% CI: 1.11-1.87). Consistent with our findings, Al-Qurayshi et al. similarly noted that private insurance status and higher income level were associated with an increased likelihood or receiving immunotherapy among patients with advanced melanoma (30). Of note, while private insurance status was associated with a higher odds of receiving immunotherapy among HBC patients on unadjusted analyses, this association did not persist once income status was considered in the multivariate model as these covariates were likely colinear. While the cost related 
to immunotherapy receipt among $\mathrm{HBC}$ patients could not be estimated in the current study, other studies have reported that the cost of immune checkpoint inhibitors for melanoma and NSCLC range from \$64,000-\$145,000 per person per year (31). A separate study noted a cost of $\$ 93,000$ per treatment for an FDA approved cancer vaccine therapy to treat prostate cancer (32). Given the high cost of immunotherapy, the health care system and insurance plans will need to take into consideration the ramifications of this therapy relative to the potential financial impact on patients.

Another interesting aspect of the current study was the characterization of the timing of immunotherapy among surgical and non-surgical patients. For most patients with advanced cancer, multi-modality therapy is required and the timing of therapy can vary depending on a number of factors. While immunotherapy can be administered as an adjuvant therapy or as palliative therapy in the case of recurrence among surgical patients, immunotherapy can be used either as a first line or second line therapy for those patients with more advanced unresectable disease (33). Data from the current study demonstrated that immunotherapy was typically delivered later in the HBC treatment course. Specifically, among surgical patients who received immunotherapy, it was delivered on average 40-50 days following surgery (Figure 3A). In contrast, among patients with more advanced disease who did not undergo surgical resection, cytotoxic chemotherapy was more commonly utilized after the diagnosis of $\mathrm{HBC}$ with immunotherapy being administered later in the clinical course (Figure $3 A, B$ ). Not surprisingly, these data suggest that immunotherapy was largely being used as second or third line agents in most patients with advanced disease after standard chemotherapy likely failed to control disease progression.

Several limitations should be considered when interpreting data in the current study. While the NCDB is a large administrative database, some of the data elements are limited including the lack of information on the possible contraindications for immunotherapy such as renal dysfunction, performance status, and patient preference. Similar to previous studies (34-38), the current study included patients with resectable tumors who had favorable prognostic features (i.e., small tumors, negative surgical margins), some of whom may not have traditionally been considered for neoadjuvant or adjuvant systemic therapy. In addition, data on the specific types of immunotherapy regimens, the number of cycles, as well as any associated toxicities among those patients receiving immunotherapy were not available. As data from NCDB were also only available until 2015, we were unable to analyze data for more recent years.

\section{Conclusions}

The overall utilization of immunotherapy in the US among patients with $\mathrm{HBC}$ was very low, yet has increased over the last several years examined. Certain socioeconomic factors were associated with an increased likely of receiving immunotherapy, which may suggest disparities in access or enrollment of patients with lower socioeconomic status. As the role of immunotherapy for HBC continues to expand, a better understanding of the overall utilization patterns, as well as the factors associated with receipt of immunotherapy, will be needed.

\section{Acknowledgments}

None.

\section{Footnote}

Conflicts of Interest: The authors have no conflicts of interest to declare.

Ethical Statement: The authors are accountable for all aspects of the work in ensuring that questions related to the accuracy or integrity of any part of the work are appropriately investigated and resolved. The study was approved by the institutional research review committee.

\section{References}

1. de Groen PC, Gores GJ, LaRusso NF, et al. Biliary tract cancers. N Engl J Med 1999;341:1368-78.

2. Spolverato G, Bagante F, Tsilimigras D, et al. Management and outcomes among patients with mixed hepatocholangiocellular carcinoma: A population-based analysis. J Surg Oncol 2019;119:278-87.

3. Ferlay J, Soerjomataram I, Dikshit R, et al. Cancer incidence and mortality worldwide: sources, methods and major patterns in GLOBOCAN 2012. Int J Cancer 2015;136:E359-86.

4. El-Serag HB, Davila JA, Petersen NJ, et al. The continuing increase in the incidence of hepatocellular carcinoma in the united states: An update. Annals of Internal Medicine 2003;139:817-23. 
5. Ryerson AB, Eheman CR, Altekruse SF, et al. Annual Report to the Nation on the Status of Cancer, 1975-2012, featuring the increasing incidence of liver cancer. Cancer 2016;122:1312-37.

6. Valle J, Wasan H, Palmer DH, et al. Cisplatin plus gemcitabine versus gemcitabine for biliary tract cancer. $\mathrm{N}$ Engl J Med 2010;362:1273-81.

7. Llovet JM, Ricci S, Mazzaferro V, et al. Sorafenib in advanced hepatocellular carcinoma. N Engl J Med 2008;359:378-90.

8. Darcy PK, Neeson P, Yong CS, et al. Manipulating immune cells for adoptive immunotherapy of cancer. Curr Opin Immunol 2014;27:46-52.

9. Xie Y, Xiang Y, Sheng J, et al. Immunotherapy for Hepatocellular Carcinoma: Current Advances and Future Expectations. J Immunol Res 2018;2018:8740976.

10. Raufi A, Tirona MT. Prospect of the use of checkpoint inhibitors in hepatocellular cancer treatments. Cancer management and research 2017;9:19-27.

11. Moris D, Rahnemai-Azar AA, Zhang X, et al. Program death-1 immune checkpoint and tumor microenvironment in malignant liver tumors. Surg Oncol 2017;26:423-30.

12. Peng BG, Liang $L J, H e$, et al. Tumor vaccine against recurrence of hepatocellular carcinoma. World J Gastroenterol 2005;11:700-4.

13. Lee WC, Wang HC, Hung CF, et al. Vaccination of advanced hepatocellular carcinoma patients with tumor lysate-pulsed dendritic cells: a clinical trial. J Immunother 2005;28:496-504.

14. Tsilimigras DI, Ntanasis-Stathopoulos I, Moris D, et al. Histone deacetylase inhibitors in hepatocellular carcinoma: A therapeutic perspective. Surg Oncol 2018;27:611-8.

15. Pardee AD, Butterfield LH. Immunotherapy of hepatocellular carcinoma: Unique challenges and clinical opportunities. Oncoimmunology 2012;1:48-55.

16. DeLeon TT, Zhou Y, Nagalo BM, et al. Novel immunotherapy strategies for hepatobiliary cancers. Immunotherapy 2018;10:1077-91.

17. Jiang Y, Li Y, Zhu B. T-cell exhaustion in the tumor microenvironment. Cell Death Dis 2015;6:e1792.

18. Trinchieri G. Cancer and inflammation: an old intuition with rapidly evolving new concepts. Annu Rev Immunol 2012;30:677-706.

19. Crespo J, Sun H, Welling TH, et al. T cell anergy, exhaustion, senescence, and stemness in the tumor microenvironment. Curr Opin Immunol 2013;25:214-21.

20. Kalathil S, Lugade AA, Miller A, et al. Higher frequencies of GARP(+)CTLA-4(+)Foxp3(+) T regulatory cells and myeloid-derived suppressor cells in hepatocellular carcinoma patients are associated with impaired T-cell functionality. Cancer Res 2013;73:2435-44.

21. Robert C, Long GV, Brady B, et al. Nivolumab in previously untreated melanoma without BRAF mutation. N Engl J Med 2015;372:320-30.

22. Brahmer J, Reckamp KL, Baas P, et al. Nivolumab versus Docetaxel in Advanced Squamous-Cell Non-Small-Cell Lung Cancer. N Engl J Med 2015;373:123-35.

23. Motzer RJ, Escudier B, McDermott DF, et al. Nivolumab versus Everolimus in Advanced Renal-Cell Carcinoma. N Engl J Med 2015;373:1803-13.

24. Balar AV, Galsky MD, Rosenberg JE, et al. Atezolizumab as first-line treatment in cisplatin-ineligible patients with locally advanced and metastatic urothelial carcinoma: a single-arm, multicentre, phase 2 trial. Lancet 2017;389:67-76.

25. Chai Y. Immunotherapy of biliary tract cancer. Tumour Biol 2016;37:2817-21.

26. El-Khoueiry AB, Sangro B, Yau T, et al. Nivolumab in patients with advanced hepatocellular carcinoma (CheckMate 040): an open-label, non-comparative, phase $1 / 2$ dose escalation and expansion trial. Lancet 2017;389:2492-502.

27. Connell CM, Raby S, Beh I, et al. Cancer immunotherapy trial registrations increase exponentially but chronic immunosuppressive glucocorticoid therapy may compromise outcomes. Ann Oncol 2017;28:1678-9.

28. Shavers VL, Brown ML. Racial and Ethnic Disparities in the Receipt of Cancer Treatment. J Natl Cancer Inst 2002;94:334-57.

29. Ward E, Jemal A, Cokkinides V, et al. Cancer Disparities by Race/Ethnicity and Socioeconomic Status. CA Cancer J Clin 2004;54:78-93.

30. Al-Qurayshi Z, Crowther JE, Hamner JB, et al. Disparities of Immunotherapy Utilization in Patients with Stage III Cutaneous Melanoma: A National Perspective. Anticancer Res 2018;38:2897-901.

31. Tartari F, Santoni M, Burattini L, et al. Economic sustainability of anti-PD-1 agents nivolumab and pembrolizumab in cancer patients: Recent insights and future challenges. Cancer Treatment Reviews 2016;48:20-4.

32. Lesterhuis WJ, Haanen JBAG, Punt CJA. Cancer immunotherapy--revisited. Nat Rev Drug Discov 2011;10:591.

33. Longo V, Gnoni A, Casadei Gardini A, et al. Immunotherapeutic approaches for hepatocellular 
carcinoma. Oncotarget 2017;8:33897-910.

34. Okusaka T, Ikeda M. Immunotherapy for hepatocellular carcinoma: current status and future perspectives. ESMO Open 2018;3:e000455.

35. Hui D, Qiang L, Jian W, et al. A randomized, controlled trial of postoperative adjuvant cytokine-induced killer cells immunotherapy after radical resection of hepatocellular carcinoma. Dig Liver Dis 2009;41:36-41.

36. Wang H, Liu A, Bo W, et al. Adjuvant immunotherapy with autologous cytokine-induced killer cells for hepatocellular carcinoma patients after curative resection, a systematic review and meta-analysis. Dig Liver Dis 2016;48:1275-82.

37. Lepisto AJ, Moser AJ, Zeh H, et al. A phase I/II study of a MUC1 peptide pulsed autologous dendritic cell vaccine as adjuvant therapy in patients with resected pancreatic and biliary tumors. Cancer Ther 2008;6:955-64.

38. Xu L, Wang J, Kim Y, et al. A randomized controlled trial on patients with or without adjuvant autologous cytokine-induced killer cells after curative resection for hepatocellular carcinoma. Oncoimmunology 2015;5:e1083671.

Cite this article as: Sahara K, Farooq SA, Tsilimigras DI, Merath K, Paredes AZ, Wu L, Mehta R, Hyer JM, Endo I, Pawlik TM. Immunotherapy utilization for hepatobiliary cancer in the United States: disparities among patients with different socioeconomic status. Hepatobiliary Surg Nutr 2020;9(1):13-24. doi: 10.21037/hbsn.2019.07.01 
Supplementary

Table S1 International Classification of Disease for Oncology (ICD-O-3) codes, Diagnoses and Procedure Codes to Identify Cases

\begin{tabular}{lll}
\hline \multirow{2}{*}{ Primary tumor } & \multicolumn{1}{c}{ ICD-O-3 code } & \multicolumn{1}{c}{ Morphological codes } \\
\cline { 2 - 3 } & \multicolumn{1}{c}{ Topographical codes } \\
\hline Hepatocellular carcinoma & C22.0 & $8170 / 3,8171 / 3,8180 / 3$ \\
Intrahepatic cholangiocarcinoma & C22.0 or C22.1 & $8970 / 3,8020 / 3,8160 / 3,8980 / 3,8963 / 3$ \\
Gallbladder cancer & C23.9 & $8140 / 3,8144 / 3,8310 / 3,8480 / 3,8490 / 3,8560 / 3,8070 / 3,8020 / 3$ \\
Extrahepatic bile duct cancer & C24.0, C24.1, C24.8, C24.9 & $8140 / 3,8144 / 3,8310 / 3,8480 / 3,8490 / 3,8560 / 3,8070 / 3,8020 / 3$ \\
\hline
\end{tabular}

